\title{
Zur Brachlegung der Monti und Alpen im Verzascatal
}

Die Bergtäler der Alpensüdseite sind zur Zeit von einer eigentlichen Wüstungswelle ergriffen. Zahlreiche Siedlungen werden verlassen; die Nutzung von Gebäuden und Flur wird aufgegeben. Nach kurzer Zeit tritt die Verbuschung und Verwaldung des Kulturlandes ein. Man kann mit HAUSER (1975) einiggehen, der diesen aktuellen Landschaftswandel mit den Wüstlegungen des Spätmittelalters direkt vergleicht. Die Folge der fortschreitenden Nutzungsauflassung ist das vermehrte Auftreten des Brachlandes' als Landschaftselement. Von der Brachlegung ist im Tessin vor allem die Monti- und Alpstufe betroffen, da sich hier der Wandel der Wirtschaftsstruktur besonders heftig bemerkbar macht. Aber auch die schlecht erschlossenen Talsiedlungen waren lange Zeit von der Abwanderung bedroht. Ein eindrückliches Beispiel hiefür ist die Heimatschutzaktion 1975 zur Rettung des Dorfes Coripo im Verzascatal, das in einzigartiger Weise die Schönheit des alten Tessiner Siedlungsbildes verkörpert.

\section{Wandel der Wirtschafts- und Sozialstruktur}

Bis zur Mitte dieses Jahrhunderts wurde die Tessiner Berglandschaft intensiv bewirtschaftet. COLOMBO (1979) benutzt zur Beschreibung der traditionellen Wirtschaftsform des Verzascatales den Begriff Autarkiekomplex. $\mathrm{Zu}$ dieser auf Selbstversorgung ausgerichteten Landwirtschaft gehörte neben der Viehzucht auch der Ackerbau, der sich an manchen Orten bis auf die Montistufe erstreckte. Davon zeugen heute noch Ackerterrassen, ummauerte Gärten und Backöfen in den Hauptsiedlungen. Für die Viehwirtschaft erschlo $B$ man die steilen Bergflanken mit Monti und Alpen, wobei zum Beispiel in Lavertezzo/Verzasca die Monti zwischen 800 und $1100 \mathrm{~m}$, die Alpgebäude zwischen 1600 und $2100 \mathrm{~m}$ liegen. Wenn man bedenkt, daß diese Wiesen und Weiden oftmals nur über lange und gefährliche Wege erreicht werden können, wird die extreme Ausnutzung des Wirtschaftsraumes bewußt. Dies zeigt sich auch in der differenzierten Struktur des traditionellen Landwirtschaftsbetriebes, der sich in der Regel über drei bis vier Höhenstufen erstreckte. Die komplizierten innerbetrieblichen Wanderungsschemen wurden im Verzascatal erstmals von GSCHWEND (1946) aufgezeichnet. Die Wirtschaftsform basierte auf der Großfamilie, da häufig auf allen Höhenstufen gleich- zeitig gearbeitet werden mußte. Die genossenschaftliche Organisation war praktisch unbekannt. In seinen Untersuchungen beschreibt COLOMBO (1979), wie seit Jahrzehnten die Komplexität dieser Betriebe aufgelöst wird, bis sich schließlich die Bewirtschaftung nur noch auf die Talstufe beschränkt. Die aktuelle Brachlegung ist unter anderem vor dem Hintergrund der Auflösung der patriarchalischen Gesellschaftsstruktur zu sehen. Die Aufsplitterung der Großfamilien, die von KAUER (1976) in seinem Roman "Spätholz» treffend geschildert wird, macht die Bewirtschaftung der zahlreichen Besitzparzellen weitgehend unmöglich.

RICHTER (1975) belegt den Rückgang der Alpwirtschaft im Verzascatal mit eindrücklichen Zahlen (Tabelle 1). Während die Zahl des gealpten Rindviehs auf ein Minimum gesunken ist, hat sich die extensive Schafhaltung parallel dazu entwickelt. Schafe werden vielfach im Nebenerwerb gehalten, und man kann sich fragen, inwieweit dies bezeichnend für auslaufende Betriebe ist. Auffällig ist die intensive Milchviehhaltung in Sonogno. Dieses Dorf liegt zuhinterst im Verzascatal und entfällt somit dem Einzugsbereich der Agglomeration Locarno. Zudem verfügt Sonogno über einen aktiven jungen Bauernstand, der durch Kooperation nach neuen Betriebsformen sucht. In den übrigen Talgemeinden hat sich seit 1971 die Situation auch weiter verändert. In Lavertezzo zum Beispiel wurde 1978 nur noch eine Alp (Corte nuovo) mit 12 GVE bestoßen. Am Beispiel der Gemeinde Lavertezzo/Verzasca sollen die landschaftlichen Veränderungen, die durch die Nutzungsauflassung in der Monti- und Alpstufe ausgelöst werden, aufgezeigt werden.

\section{Landschaftswandel in Lavertezzo/Verzasca}

Die Gemeinde Lavertezzo ${ }^{2}$ liegt im mittleren Abschnitt des Verzascatals, der durch seine steile Topographie charakterisiert ist. Das 5813 ha große Gemeindegebiet erstreckt sich von $530 \mathrm{~m}$ bis $2500 \mathrm{~m}$ und umfaßt die 3 Täler Agro, Carecchio und Pincascia, die als Lavertezzotäler bekannt sind. In diesen Tälern liegen, zum Teil weit von der Hauptsiedlung entfernt, die Monti und Alpen. Da sich auf Talstufe

Pierre Walther, Assistent am Geographischen Institut der Universität Zürich, Blümlisalpstraße 10, 8006 Zürich. 
Tabelle 1: Gealpte Viehbestände und bestoßene Alpen (in Klammern) im Verzascatal nach RICHTER (1975)

\begin{tabular}{|c|c|c|c|c|c|c|c|c|}
\hline \multirow[b]{3}{*}{ Vogorno } & \multicolumn{2}{|c|}{ Milchkühe } & \multicolumn{2}{|l|}{ Rinder } & \multicolumn{2}{|l|}{ Ziegen } & \multicolumn{2}{|l|}{ Schafe } \\
\hline & 1920 & 1971 & 1920 & 1971 & 1920 & 1971 & 1911 & 1971 \\
\hline & $178(7)$ & $0(0)$ & $58(7)$ & 19 (1) & $505(7)$ & $0(0)$ & $120(2)$ & $200(1)$ \\
\hline Lavertezzo & $235(12)$ & $65(3)$ & $85(11)$ & $20(3)$ & $806(12)$ & $133(3)$ & $550(5)$ & 450 (3) \\
\hline Brione & $169(8)$ & $0(0)$ & $69(8)$ & $0(0)$ & $489(8)$ & $20(1)$ & $230(3)$ & $550(1)$ \\
\hline Coripo & $7(1)$ & $0(0)$ & $3(1)$ & $0(0)$ & $20(1)$ & $0(0)$ & $0(0)$ & $0(0)$ \\
\hline Gerra Verzasca & $66(2)$ & $0(0)$ & $21(1)$ & $0(0)$ & $35(2)$ & $0(0)$ & $50(1)$ & $400(2)$ \\
\hline Frasco & $61(4)$ & $0(0)$ & $23(4)$ & $0(0)$ & $160(4)$ & $0(0)$ & $245(3)$ & $150(1)$ \\
\hline Sonogno & $106(4)$ & 97 (3) & $20(4)$ & $103(4)$ & $260(4)$ & 305 (3) & $30(1)$ & $50(1)$ \\
\hline
\end{tabular}

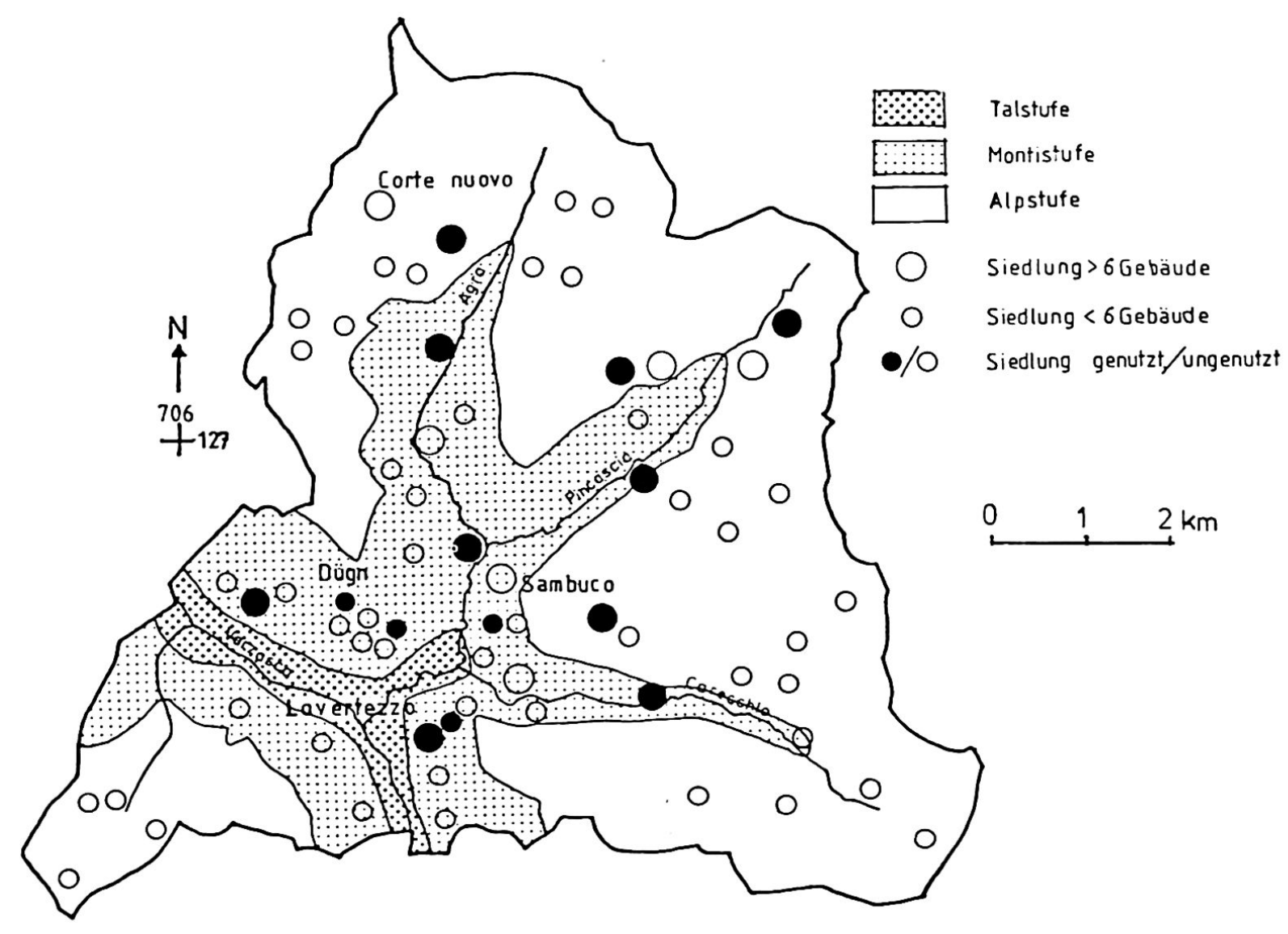

Figur 1 Genutzte und verlassene Siedlungen in der Monti- und Alpstufe in Lavertezzo/Verzasca (1979).

praktisch keine natürlichen Terrassen für Wiesland befinden, kommt diesen höheren Wirtschaftsstufen seit jeher für die Landwirtschaft eine besondere Bedeutung zu. Dabei war man auch bereit, täglich große Distanzen zu Fuß zurückzulegen. Die Alp Corte nuovo $(1750 \mathrm{~m})$, die im ALPKATASTER 1911 noch als eine der schönsten und wichtigsten Alpen des Verzascatales beschrieben wird, ist von der Talsiedlung Lavertezzo/Chiesa $(550 \mathrm{~m})$ nur über eine vierstündige Wegstrecke erreichbar. Der größte Teil der Monti und
Alpen befindet sich auf den schmalen Hangleisten, die von GYGAX (1934/35) als alte Talböden interpretiert werden.

Figur 1 zeigt den funktionalen Wandel der Alpwirtschaft in Lavertezzo. Die Karte bezieht sich auf die Gebäudenutzung, da die Flächenbewirtschaftung wegen der extensiven Schaf- und Ziegenhaltung nur schwer erfaßbar ist. In der Kategorie "genutzt» eingeschlossen ist auch die Verwendung der Häuser als Wochenend- oder Jagdunterkunft. Zu Beginn dieses 


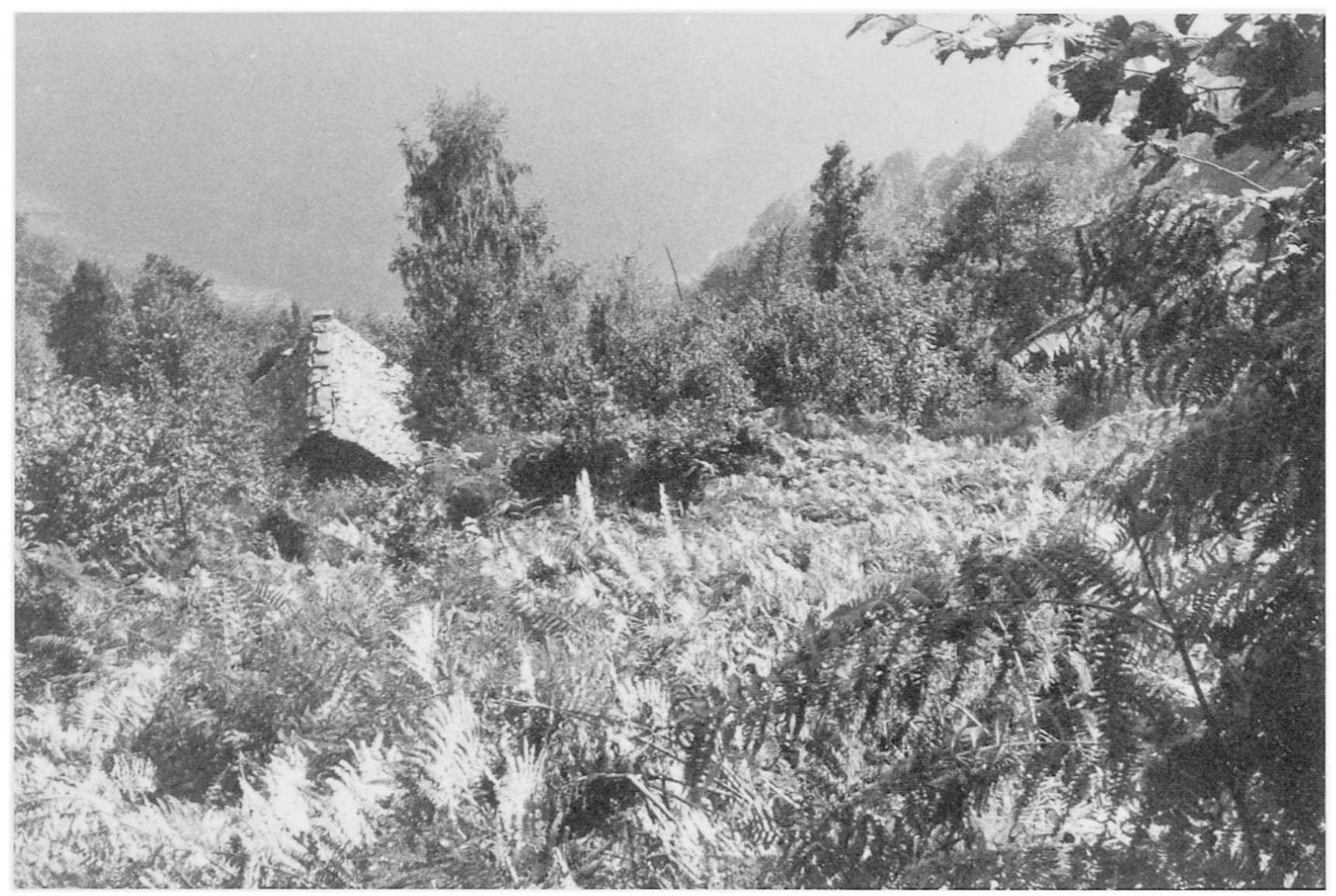

Bild 1 Ausgedehnte Flächen von pteridium aquilinum (Adlerfarn) in Dugn (707 750/125 000) (September 1978).

Jahrhunderts dienten noch alle Siedlungen der Landwirtschaft. Heute werden nur noch einige größere, gut begehbare Häusergruppen von Einheimischen in Stand gehalten. Die übrigen Siedlungen, vor allem die kleinen, zerfallen.

Nach der Nutzungsauflassung tritt rasch eine Veränderung der Vegetation ein. Als erstes ändert sich die botanische Zusammensetzung des Grünlandes. SURBER (1973) spricht von einer Gras-/Krautphase. Sie ist meistens mit einer starken Zunahme der Artenzahl im Pflanzenbestand verbunden. Vor allem die ausläuferbildenden Pflanzen, die durch die Nutzung unterdrückt werden, vermehren sich rasch. Physiognomisch tritt dieses erste Stadium, wie auch die von SURBER (1973) aufgeführte Staudenphase, in der Tessiner Berglandschaft vor allem an trockenen, sonnenexponierten Hängen in Erscheinung. Dort bilden oftmals große Flächen von pteridium aquilinum (Bild 1) jahrzehntelang eine wirksame Barriere gegen die Verbuschung. Durch das erste Auftreten von Holzgewächsen wird die Brachfläche in das Stadium der Strauchphase geführt. Mit der Bildung einer Buschvegetation ist eine Rückführung in Landwirtschaftliches Kulturland praktisch undenkbar geworden. Einerseits fält die Parzelle bereits unter das Forstgeset $^{3}$, das heute allerdings in der Praxis nicht mehr so streng gehandhabt wird (WULLSCHLEGER 1976); andererseits ist das Roden der Büsche mit einem erheblichen Sonderaufwand verbunden, und auch HELBLING (1975, S. 24) meint, daß selbst kaum noch Optimisten daran denken, die einmal verbuschten Flächen in Krisenzeiten wieder in Kultur zu nehmen. Mit dem Aufwachsen der Büsche wird das Brachland schließlich über die Baumphase in einen gleichförmigen Sekundärwald zurückgeführt.

Die Dynamik dieser Vegetationssukzession ist von zahlreichen Faktoren abhängig und erst allmählich in Regeln faßbar. Pflanzensoziologische Untersuchungen haben gezeigt (KTBL 1977, ALTHER 1978), daß Faktoren wie Bewirtschaftungsart vor der Brachlegung (Endbewirtschaftung), Exposition, Lückigkeit des Ausgangsbestandes und Höhenlage eine große Rolle spielen. Sicher kann man feststellen, daß der Sukzessionsprozeß in den Tälern der Alpensüdseite im Vergleich zu anderen Regionen des Alpenraumes rascher verläuft. Dies hat verschiedene Gründe: einerseits ist das insubrische Klima der Südschweiz mit seiner hohen Sonneneinstrahlung und Luftfeuchtigkeit für das Pflanzenwachstum günstig; andererseit bietet der steinige Boden für die jungen Holzgewächse genügend Nischen zum ungestörten Wachstum. Ein gutes Beispiel ist der im Tessin weit verbreitete Corylus-Buschwald, der sich als Sekundärwald auf steinigen Brachflächen in mittlerer Höhenlage rasch entwickelt. Dabei kann man feststellen, daß die Haselbüsche meist im Schutze von Steinen aufwachsen. 
Ein wichtiger Faktor für den Sukzessionsverlauf ist die Vegetationszusammensetzung der näheren Umgebung der Brachfläche. Mit den Jahren gleicht sich in der Regel die Brachlandvegetation dem angrenzenden Pflanzenbestand an. Windfrüchter wie acer, fraxinus und betula nehmen die aufgelassenen Flächen schnell in Besitz, während sich castanea usw. langsamer verbreitet. Ein eindrückliches Beispiel ist die Verwaldung mit acer pseudoplatanus in der Montistufe, wo in der Umgebung der Gebäude seit jeher mächtige Ahornbäume als Schattenspender gepflegt wurden. Diese Bäume verbreiten ihre Ableger im Umkreis von 20-30 m, so daß nach der Brachlegung vor allem an Schattenhängen in mittlerer Höhenlage in wenigen Jahren ein dichter Ahorn-Sekundär-Wald aufwächst.

Die Entwicklung der Brachlandvegetation wird ebenfalls von hydrologischen Faktoren gelenkt. Auf der Alpstufe bildet alnus viridis entlang von Bächen und Wassergräben einen gleichförmigen Buschbestand. Die Untersuchungen von KOERNER ET AL. (1978) zeigen, daß die Alpenerle für etwa 60 Jahre eine stabile Vegetationsgesellschaft bildet. Die Verbuschung der Alpflächen mit Zwergsträuchern wie rhododendron ferrugineum, juniperus nana und vaccinium L. wird vielerorts als eine bedrohliche Entwicklung betrachtet. Daneben ist im Verzascatal in dieser Höhenlage auch die Verwaldung mit larix sehr aktiv. Am Beispiel dieser Lärchenwälder zeigt sich auch, daß Brachlandvegetation durchaus ihre Reize und einen hohen ökologischen Wert hat. Untersuchungen aus Deutschland bestätigen die Zunahme der Fauna auf Brachland (KTBL, 1977).

\section{Ein Beispiel: Monte Sambuco (Lavertezzo)}

Welches Ausmaß diese Landschaftsveränderungen im Tessin einnehmen, läßt sich am Beispiel des Monte Sambuco (709 200/125 500) zeigen (Bild 2). Das Monte liegt, wie Figur 1 zeigt, auf $800 \mathrm{~m}$ an einem schattigen, westexponierten Steilhang. Die Siedlung gliedert sich in 4 Häusergruppen, wovon die größte 16 Gebäude umfaßt. Neben 16 mächtigen Stallscheunen stehen in Sambuco 6 Wohhäuser und 2 Nebengebäude; 5 Häuser sind schon heute bis auf die Grundmauern zerfallen. Da der steile Hang keine natürliche Hangleiste bildet, ist die Siedlung terrassenartig an die Bergflanke
$2 a$

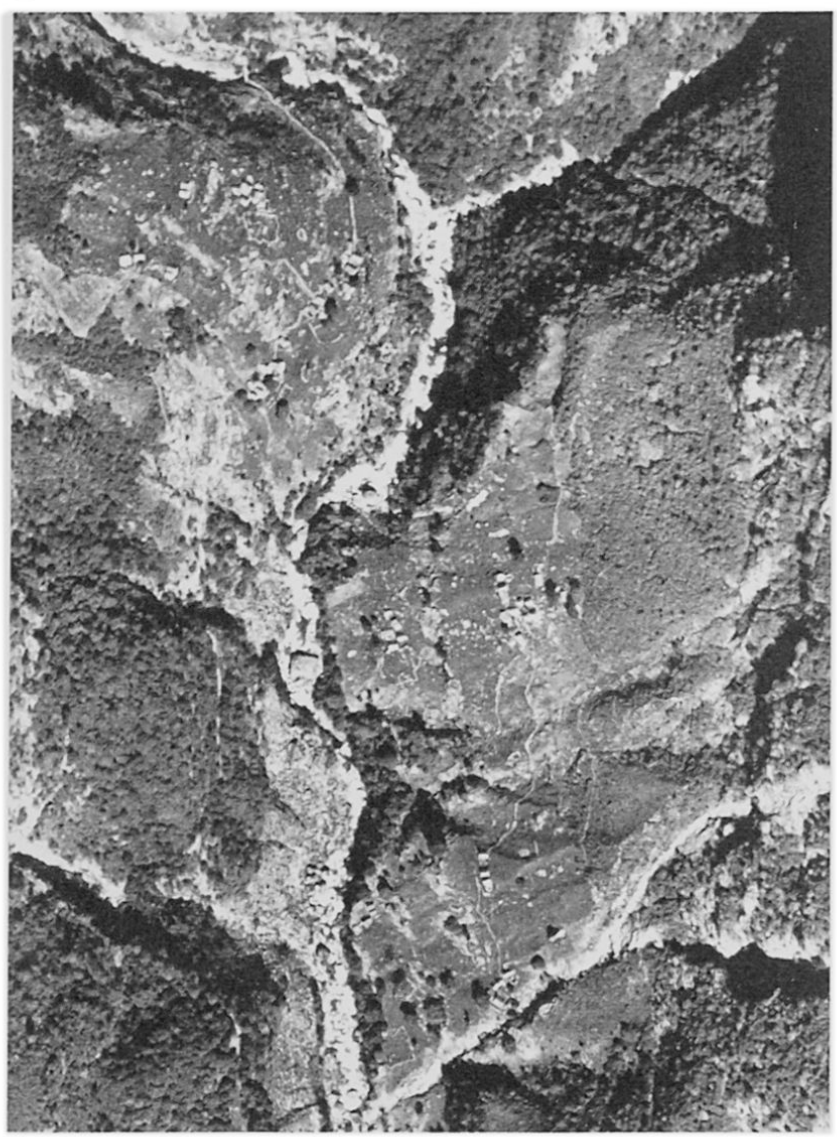

$2 \mathrm{~b}$

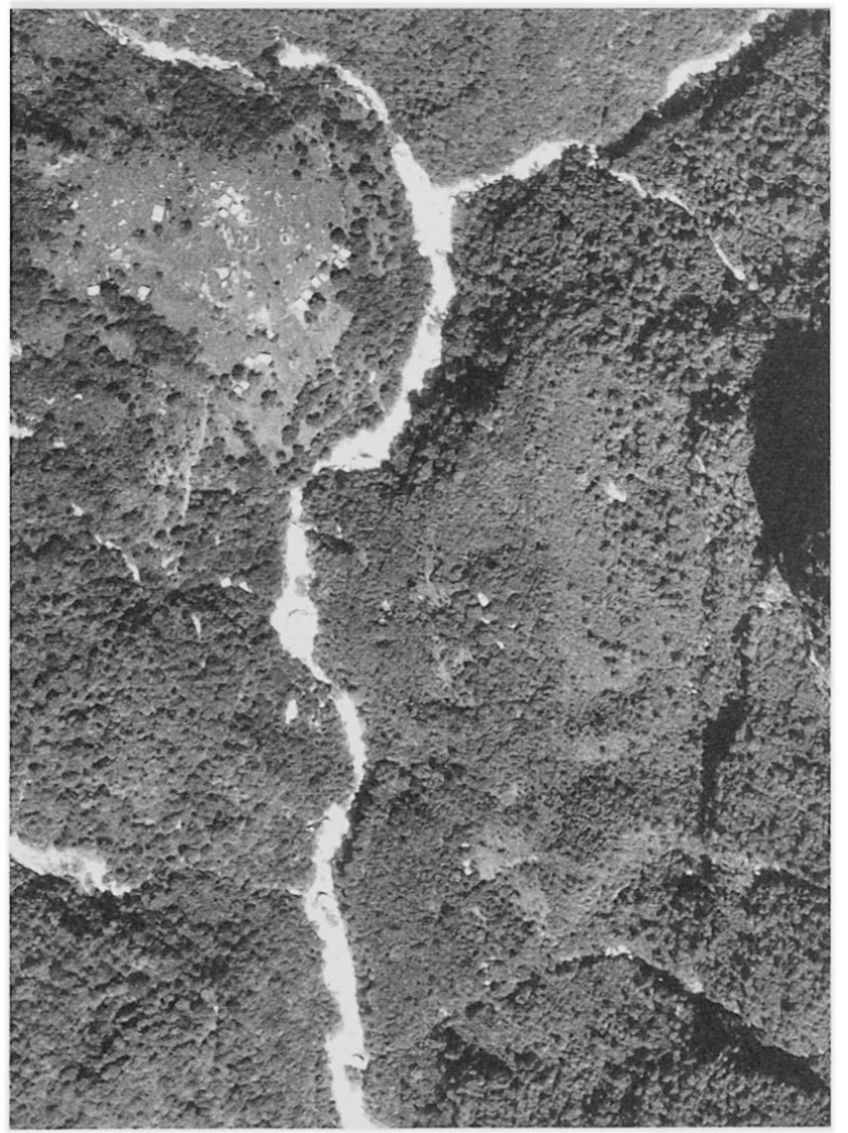

Bild 2 Verwaldung von Monte Sambuco/Lavertezzo (709 200/125 500)

Bild 2a (links): Monte Sambuco Oktober 1945 (Maßstab ca. 1:7000)

Bild 2b (rechts): Monte Sambuco Juli 1977 (Maßstab ca. 1:7000)

Reproduziert mit Bewilligung der Eidg. Landestopographie 
angebaut. Nach Auskunft des LANDWIRTSCHAFTLICHEN PRODUKTIONSKATASTERS (1945) wurde Sambuco früher intensiv mit Fettwiesen und Weiden genutzt. Schon 1945 beklagte man sich über die geringe Mächtigkeit und den großen Steingehalt des Bodens.

Bild $2 a$ zeigt Monte Sambuco im Jahre 1945. Das funktionale Zentrum der Siedlung mit Dorfplatz und Bildstock befand sich in der größten Häusergruppe. Das Luftbild zeigt, daß die Wiesen, obwohl stark mit Steinen durchsetzt, noch intensiv bewirtschaftet wurden. An der steilen Flanke oberhalb der Siedlung hat sich bereits ein junger ${ }^{4}$, dichter Corylus-Buschwald ausgebreitet, was auf eine Nutzungsextensivierung zu Beginn dieses Jahrhunderts schließen läßt. Deutlich sichtbar sind auch die vereinzelten hohen Bäume im offenen Wiesland, die als Fruchtbäume (juglans regia ist in dieser Höhenlage häufig) oder als Schattenspender und Weidbäume gepflegt wurden. Ein Ausdruck der landwirtschaftlichen Nutzung sind auch die ummauerten Viehpferche im unteren Teil der Siedlung und das feingegliederte Wegsystem. 1945 ist zwischen den beiden Monti Sambuco (rechts im Bild) und Forno (links im Bild) kein Unterschied in der Bewirtschaftung ersichtlich.

Nach Auskunft einheimischer Bauern wurde Sambuco bis etwa 1960 regelmäßig bestoßen. Anschließend wurde die Nutzung aufgegeben, wobei die Brachlegung wegen der Privatbesitzverhältnisse natürlich schrittweise erfolgte. Heute wird das Monte zeitweise noch von Schafen, seit 1979 auch wieder von Ziegen beweidet.

Bild $2 b$ zeigt Monte Sambuco im Jahre 1977. Praktisch die ganze Siedlung ist verwaldet. Die Häuser sind im Luftbild kaum mehr sichtbar, weil sich um die Gebäude und entlang der Bachrunsen ein hoher Eschenwald gebildet hat. Fraxinus findet an diesem schattigen Hang einen geeigneten Standort für ein rasches Wachstum. Als Windfrüchter besetzt es die Brachflächen sehr rasch. Der Corylus-Buschwald bildet mit seinem üppigen Wuchs eine Barriere gegen das Vordringen des Hochwaldes. Die 4-5 m hohen Haselstauden dienten früher als Ziegenweide, haben aber heute keinen wirtschaftlichen Nutzen mehr. Zudem wird das Wegsystem von den Wurzeln der Bäume angegriffen, was schließlich Sambuco in naher Zukunft unbegehbar machen wird. Welche Bedeutung der Faktor Erschließung bei der Brachlegung einnimmt, ist aus der unterschiedlichen Entwicklung von Sambuco und Forno ersichtlich. Monte Forno liegt an einem gut begehbaren Weg und ist auch heute noch landwirtschaftlich genutzt, während Sambuco aufgelassen ist.

\section{Folgerungen}

Am Beispiel von Monte Sambuco sieht man, welch tiefgreifende landschaftliche Veränderungen durch die Brachlegung in den Tessiner Alpentälern ausge- löst werden. Die rasche Zunahme der Waldfläche beeinflußt den ökologischen Landschaftshaushalt. Die Kulturlandschaft zerfällt.

Aus planerischer Sicht wäre es interessant, diesen Wandel festzuhalten. Brachlandkarten sind für eine Forstplanung unerläßlich. Es stellt sich auch die Frage, welche Bedeutung diesen Kulturlandverlusten im gesamtwirtschaftlichen Konzept - vor allem im Hinblick auf eine Nahrungsmittelversorgung in Krisenzeiten - beizumessen ist. Das Beispiel Sambuco zeigt auch, daß im Tessiner Berggebiet vor allem ein Problem Beachtung verdient: Viele schützenswerte Siedlungen zerfallen heute im Sekundärwald. Eine Siedlungsplanung ist in diesem Bereich dringend nötig. SUFFERT (1978) hat in seiner "carte éco-agrologique des Pyrénées» die Landnutzungskategorie "éspaces marginalisés ou abandonnés» (Brachland) im Berggebiet der Pyrenäen großflächig aufgenommen. Das Brachland steht als Einheit gleichwertig neben Wald und Kulturland. Die Grundlagen sammelte er mit Luftbildinterpretation, Feld- und Archivarbeit. Aus dieser Arbeit ersehen wir das Hauptziel einer Brachlandkartierung: Versachlichung der vieldiskutierten Brachlandthematik im regionalen Vergleich. Eine solche kartographische Aufnahme des aktuellen Landschaftswandels ist in den Tessiner Alpentälern nötiger denn je.

\section{Anmerkungen}

Anm. 1:Im Bewußtsein der lebhaften Begriffsdiskussion wähle ich den Ausdruck «Brachland». Darunter verstehe ich «nicht mehr genutztes Landwirtschaftliches Kulturland, das noch nicht zu Sekundärwald (Def. nach Landesforstinventar) geworden ist».

Anm. 2: Ein weiterer Teil des Gemeindegebietes befindet sich in der Magadinoebene (Lavertezzo/Piano).

Anm. 3: Vollziehungsverordnung zum Bundesgesetz betreffend die eidg. Oberaufsicht über die Forstpolizei (1. 10. 1965), Art. 1: «Als Wald im Sinne des Gesetzes gilt, ... jede mit Waldbäumen oder -sträuchern bestockte Fläche ....».

Anm. 4: Das Alter der Corylus-Vegetation wurde 1978 auf etwa 40 Jahre bestimmt.

\section{Zitierte Literatur}

ALPKATASTER, Schweizerisches. Kt. Tessin. Bern 1911, 1976.

ALTHER, E. und STÄHLIN, A.: Entwicklung von Böden und Pflanzenbeständen auf Brachland und ihre Dynamik während 150 Jahren. In: Das wirtschaftseigene Futter, Frankfurt 1977.

ColomBo, D.: Die Alpwirtschaft des Val Verzasca. In: Die Alpen, Zeitschrift des Schweizer Alpenclubs, 1979/3. 
GSChwend, M.: Das Val Verzasca. Aarau 1946.

GYGAX, F.: Beitrag zur Morphologie des Verzascatales. In: Der Schweizer Geograph, Zürich 1934/35.

HAUSER, A.: Brachland oder Wüstung? In: Schweizerische Zeitschrift für Forstwesen, 1975/1.

HELBLING, F.: In: GRUBINGER, J.: Technische und wirtschaftliche Aspekte der Nutzung von Böden im Grenzertragsbereich. Tänikon 1975.

KAUER, w.: Spätholz. Zürich 1976.

KOERNER, C. ET AL.: Wachstumsdynamik von Grünerlen auf ehemaligen Almflächen an der zentralalpinen Waldgrenze. In: CERNUSCA, A.: Ökologische Analysen von Almflächen im Gasteiner Tal. Innsbruck 1978.

KTBL, BIERHALS ET AL.: Brachflächen in der Landschaft. Darmstadt 1976.

\section{Literaturbesprechung}

LASCHINGER, Werner / LOETSCHER, Lienhard: Basel als urbaner Lebensraum. Basler Beiträge zur Geographie, Heft 22/23, 338 S., div. Abb., Selbstverlag der Universität Base1, 1978. Fr. 24.--

Als erstes darf man den beiden Verfassern sicher dazu gratulieren, dass sie das Wagnis einer Gemeinschaftsarbeit für eine Dissertation eingegangen sind. Der Erfolg hat ihnen im nachhinein recht gegeben. Der vorliegende Band unfasst einen gemeinsamen theoretischen Teil, sowie zwei

Fallstudien, welche von den Verfassern gesondert erarbeitet und vorgestellt wurden (um den Fakultätsansprüchen an eine Dissertation zu genügen!)

Ausgehend von den bisherigen sozialgeographischen Ansätzen zur Erfassung räumlich sich auswirkender Prozesse entwickeln die Verfasser unter Einbezug der Systemtheorie ein Mode11 des urbanen Systems. Verschiedene, schon bisher gebrauchte Begriffe werden dabei kritisch unter die Lupe genommen und präziser definiert, andere werden durch Neuschöpfungen ersetzt. Besonders interessant sind etwa die Ausführungen über die "raumwirksamen Gruppen". Im Rahmen einer Buchanzeige kann das erarbeitete Modell auch nicht an-
LANDWIRTSCHAFTLICHER PRODUKTIONSKATASTER, Gmde. Lavertezzo. Bern 1945.

RICHTER, M.: La regressione dell'alpicoltura in Val Verzasca. Archivo storico, Bellinzona 1975.

SUFFERT, C.: Les grandes unités éco-agrologiques des Pyrénées françaises en 1970-1978. In: Revue geogr. des pyrénées et du sud-ouest. Toulouse 1978/4.

SURBER, E. ET AL.: Das Brachlandproblem in der Schweiz. Bericht EAFV Nr. 112. Birmensdorf 1974. WALTHER, P.: Art und Umfang des Dauerbrachlandes in Tessiner Alpentälern. Dipl. Arb. Geogr. Inst. Universität Zürich 1979.

WULlSCHLEGER, E.: Der Waldbegriff in der forstlichen Literatur und Gesetzgebung. Bericht EAFV Nr. 167. Birmensdorf 1976. nähernd wiedergegeben werden. Es ist aber zweifellos ein Beitrag zur theoretischen Diskussion über die Sozialgeographie des städtischen Raumes, um den zukünftige Arbeiten nicht herumkommen werden.

Das Modell wird in den Teilen 2 und 3 der Arbeit an zwei Fallstudien aus dem Raume Basel getestet: Matthäus-Quartier im Kleinbasel und Verstädterungsband Birsfelden - Rheinfelden. Die Untersuchungsgebiete ergänzen sich in ihrer Gegensätzlichkeit sehr gut und zeigen die vielfältigen Prozesse, welche sich in einer städtischen Agglomeration abspielen und meistens zu einer funktionalen Entmischung hintendieren. Dieses Buch ist für den weniger mit der Systemtheorie vertrauten Leser keine leichte Lektüre. Wer sich aber die Mühe nimmt und sich ein wenig einliest, der wird zahlreiche Denkanstösse zur vielbeklagten "Krise der Stadt" erhalten. Und letztlich hat eine solche Arbeit ja nur einen Sinn, wenn ihre Erkenntnisse von weiteren Kreisen aufgenommen und in die Gestaltung der Umwelt eingebaut werden.

GH $1980 / 1$ Jürg Rohner, Basel 